\title{
Desenvolvimento de um material educativo para a prevenção e o tratamento das lesões por fricção
}

\author{
Development of educational material for the prevention and treatment of \\ friction injuries
}
Desarrollo de material educativo para la prevención y tratamiento de lesiones por fricción

\author{
Geraldo Magela Salomé1,*
}

ORCID IDs

Salomé GM (D) https://orcid.org/0000-0002-7315-4866

\section{COMO CITAR}

Salomé GM. Desenvolvimento de um material educativo para a prevenção e o tratamento das lesões por fricção. ESTIMA, Braz. J. Enterostomal Ther., 2020, 18: e3220. https://doi.org/10.30886/ estima.v18.923_PT

\begin{abstract}
RESUMO
Objetivo: desenvolver e validar uma cartilha educativa para ajudar o profissional da saúde a prevenir e tratar da lesão por fricção. Método: estudo metodológico. Foi realizada revisão integrativa da literatura dos periódicos publicados entre 2009 e 2019 nas principais bases de dados de Ciências da Saúde. A avaliação da cartilha educativa foi conduzida com 32 enfermeiros, utilizando a técnica de Delphi. A análise estatística utilizada foi o Índice de Validade de Conteúdo. Resultados: os juízes consideraram o conteúdo da cartilha educativa entre os conceitos "inadequado" e "adequado", na primeira avaliação; após as correções solicitadas pelos juízes, a cartilha foi reenviada aos avaliadores e o conteúdo da cartilha educativa foi avaliado entre "adequado" e "totalmente adequado". O Índice de Validade de Conteúdo geral foi de 0,8118 na primeira avaliação e 1,0 na segunda avaliação. Conclusão: após a revisão integrativa da literatura, o material foi construído e validado por profissionais com experiência em avaliar e tratar de indivíduos com feridas, obtendo consenso entre os avaliadores na segunda avaliação.
\end{abstract}

DESCRITORES: Fricção; Ferimentos e lesões; Avaliação em enfermagem; Educação em saúde; Estomaterapia.

\footnotetext{
1. Universidade do Vale do Sapucaí - Programa de Mestrado Profissional em Ciências Aplicadas à Saúde - Pouso Alegre (MG), Brasil.
}

*Autor correspondente: salomereiki@univas.edu.br

Recebido: Jul. 23, 2020 | Aceito: Nov. 20, 2020 


\begin{abstract}
Objective: to develop and validate an educational booklet to help health professionals to prevent and treat friction injuries. Method: methodological study. An integrative review of the literature of journals published between 2009 and 2019 was carried out in the main databases of Health Sciences. The evaluation of the educational booklet was conducted with 32 nurses, using the Delphi technique. The statistical analysis used was the Content Validity Index. Results: the judges considered the content of the educational booklet between the concepts "inadequate" and "adequate", in the first assessment; after the corrections requested by the judges, the booklet was forwarded to the evaluators and the content of the educational booklet was rated between "adequate" and "totally adequate". The overall Content Validity Index was 0.8118 in the first assessment and 1.0 in the second assessment. Conclusion: after the integrative literature review, the material was constructed and validated by professionals with experience in evaluating and treating individuals with wounds, obtaining consensus among the evaluators in the second evaluation.
\end{abstract}

DESCRIPTORS: Friction; Wounds and injuries; Nursing assessment; Health education; Stomatherapy.

\title{
RESUMEN
}

Objetivo: Desarrollar y validar una cartilla educativa para ayudar al profesional de la salud a prevenir y tratar lesiones por fricción. Método: estudio metodológico. Fue realizada revisión integradora de la literatura de los periódicos publicados entre 2009 y 2019 en las principales bases de datos de Ciencias de la Salud. La evaluación de la cartilla educativa fue realizada con 32 enfermeros, utilizando la técnica de Delphi. El análisis estadístico utilizada fue el Índice de Validez de Contenido. Resultados: los jueces consideraron el contenido de la cartilla educativa entre los conceptos «inadecuado» y «adecuado», en la primera evaluación; luego de las correcciones solicitadas por los jueces, la cartilla fue reenviada a los evaluadores y el contenido de la cartilla educativa fue evaluado entre «adecuado» y «totalmente adecuado». El Índice de Validez de Contenido general fue de 0,8118 en la primera evaluación y 1,0 en la segunda evaluación. Conclusión: luego de la revisión integradora de la literatura, el material fue construido y validado por profesionales con experiencia en evaluar y tratar individuos con heridas, obteniendo consenso entre los evaluadores en su segunda evaluación.

DESCRIPTORES: Fricción; Heridas y lesiones; Evaluación en enfermería; Educación en salud; Estomaterapia.

\section{INTRODUÇÃO}

O tegumento é o maior órgão do corpo, necessário para a sobrevivência do ser humano e para o equilíbrio perfeito fisiológico do organismo, podendo sofrer agressões por fatores patológicos intrínsecos e extrínsecos. Essas agressões podem causar modificações na sua constituição, como queimaduras, úlceras traumáticas, dermatite, lesão por fricção (LF) e outras, podendo levar à incapacidade funcional do indivíduo e provocar alterações na sua qualidade de vida ${ }^{1-3}$.

O profissional que presta assistência aos indivíduos que apresentam fatores de risco ou que contraíram LF tem um papel importante no cuidado holístico como também desempenha trabalho de extrema relevância na prevenção e tratamento da $\mathrm{LF}^{4,5}$.

A LF advém de contusão, seja por atrito, trauma ou cisalhamento da pele. A tensão presente na retração, fricção ou choque entre a pele da pessoa e a superfície do leito ou de materiais ao redor pode provocar lesões de espessura parcial ou de espessura total ${ }^{6-9}$.

A topografia corporal mais é atingida pela LF é o dorso das mãos, os braços, os cotovelos e as pernas de pessoas idosas ou muito jovens como neonatos. A produção de exsudato seroso, especialmente nas primeiras 24 horas, torna as LF majoritariamente úmidas ${ }^{1-4}$.

Vários estudos relatam que $42 \%$ das LF estão localizadas nos cotovelos, $22 \%$ nas pernas e $13 \%$ nas mãos ${ }^{1-8}$.

Por essa razão, o enfermeiro deve avaliar a pele do paciente no momento da sua admissão e, a partir de então, diariamente; também deve orientar os profissionais da saúde e cuidadores que assistem esses indivíduos no sentido de manter a pele seca e limpa por meio da manutenção, evitando a ocorrência de cisalhamento e, consequentemente, a LF ${ }^{9,10}$.

Essas orientações podem ser feitas de forma oral ou escrita. Também é importante a elaboração de material educativo como algoritmos, cartilhas, protocolos, aplicativos, cursos online, entre outros sobre as medidas preventivas e as condutas terapêuticas ${ }^{11}$.

A construção de protocolos, manuais, cartilhas, algoritmos, guias e diretrizes que contemplam as melhores evidências científicas pode favorecer a prestação da assistência baseada em boas práticas clínicas, promovendo a melhora/recuperação da saúde antes de o indivíduo ter sido afetado ${ }^{11-13}$.

A validação de uma cartilha é um processo importante, pois determina se o instrumento é adequado para fornecer medidas analíticas e informações apropriadas para um determinado objetivo e contexto, como a avaliação da 
presença de fatores de risco para LF, diretrizes para a prevenção, tratamento dessa condição, entre outros fatores. As cartilhas validadas possibilitam um cuidado individualizado e sistematizado e a otimização de tomadas de decisão, que têm como consequência a redução de custos com os cuidados prestados pelos serviços de saúde e a prestação de assistência com o mínimo risco possível, sem danos, enfim, um cuidado com qualidade e segurança.

Este estudo teve como objetivo elaborar e validar o conteúdo de uma cartilha para prevenção e tratamento da LF. Essa cartilha visa fornecer subsídios aos profissionais de saúde que prestam assistência a indivíduos com LF ou em risco, para a elaboração e o desenvolvimento de planos de cuidado da lesão, com estratégias adequadas de prevenção e tratamento, reunindo uma conduta terapêutica com variedade de métodos propícios para executá-la.

\section{OBJETIVO}

Desenvolver e validar uma cartilha educativa para ajudar o profissional da saúde a prevenir e tratar da LF.

\section{MÉTODO}

Trata-se de estudo metodológico de produção e validação de tecnologia educativa.

O processo de construção da cartilha educativa teve a elaboração da primeira versão entre os meses de março e agosto de 2019. Após avaliação da cartilha pelos juízes, foram feitas várias alterações pelos autores, tendo sido a versão definitiva concluída entre novembro de 2019 e março de 2020. O conteúdo foi alinhado às premissas para a construção de materiais educativos de orientação para o cuidado em saúde. A elaboração do material percorreu as seguintes fases: diagnóstico situacional, revisão integrativa da literatura, formulação e montagem da cartilha educativa e sua validação e implementação ${ }^{11,12}$.

\section{Primeira fase: diagnóstico situacional}

A ideia de escrever a cartilha nasceu a partir de observações feitas durante a prática clínica dos pesquisadores, em que foi percebida, com frequência, a dificuldade dos profissionais de enfermagem em realizar exame físico, prescrever ações preventivas e tratar paciente que apresenta fatores de risco ou que já tenha sido acometido pela LF.

Se tais procedimentos não forem executados corretamente, o profissional poderá colocar o cliente em risco, com possibilidade de danos e eventos inesperados.

Apesar de as Unidades Básicas de Saúde (UBS), ambulatórios de feridas e os hospitais contarem com serviço especializado de cuidados em lesões cutâneas e seguirem protocolos descritos sobre vários procedimentos, nem sempre esses estão sendo executados adequadamente ${ }^{11,12}$. Optou-se, então, por proceder-se a uma revisão integrativa da literatura com o objetivo de identificar publicações relacionadas ao tema prevenção e tratamento de LF.

\section{Segunda fase: levantamento do conteúdo}

Realizou-se uma revisão integrativa da literatura. Delimitaram-se as seguintes etapas para o desenvolvimento da pesquisa: identificação do tema e seleção da questão de pesquisa; estabelecimento de critérios para a inclusão e exclusão de estudos; definição das informações a serem extraídas dos estudos selecionados e categorização dos estudos; avaliação dos estudos incluídos na revisão integrativa; interpretação dos resultados, apresentação da revisão; e síntese do conhecimento ${ }^{13}$.

Foi determinado como tema "cartilha educativa para prevenir e tratar da LF".

Objetivou-se responder à seguinte questão norteadora: a cartilha educativa desenvolvida apresenta qualidade satisfatória para o uso na prática clínica do profissional enfermeiro? A cartilha educativa construída neste estudo fornecerá subsídio para o profissional da saúde desenvolver medidas preventivas e conduta terapêutica para o paciente que apresenta fatores de risco ou já contraiu a LF?

Para a construção da pergunta adequada para a resolução da questão clínica pesquisada, utilizou-se a estratégia PICO14, com "P" correspondendo à população (indivíduo que apresenta fatores de risco ou contraiu LF), "I" à intervenção (prevenir e tratar LF), "C" à comparação (não se aplica, pois esse não é um estudo comparativo) e "O" correspondendo ao desfecho (cartilha educativa).

A revisão integrativa da literatura deu-se por meio das bases de dados Literatura Latino-Americana e do Caribe em Ciências da Saúde (LILACS), Medical Literature Analysis 
and Retrieval System Online (MEDLINE), Cumulative Index to Nursing and Allied Health Literature (CINAHL) e portal Scientific Electronic Library Online (SciElo). Para a seleção dos artigos foram utilizados os Descritores em Ciências da Saúde (DeCS) e o Medical Subject Headings, com as palavras-chave controladas: Fricção; Ferimentos e lesões; e Cuidados de enfermagem, associados entre si utilizando o operador booleano AND.

Para a seleção das publicações foram adotados como critérios de inclusão: apenas estudos primários que tenham ligação direta com a temática, estar disponível na íntegra, artigos originais e publicados entre 2015 e 2020.

Como critérios de exclusão: teses, dissertações, monografias, relatórios técnicos e artigos que, após leitura do resumo, não se relacionam com o objeto de estudo proposto, além das publicações que se repetiram nas bases de dados.

Fez-se a leitura dos títulos e dos resumos de forma independente entre dois autores para assegurar que os textos contemplavam a pergunta norteadora da revisão e atendiam aos critérios de inclusão estabelecidos. Em caso de dúvida a respeito da seleção, optou-se por incluir inicialmente a publicação e decidir sobre sua seleção somente após a leitura na íntegra de seu conteúdo.

Para classificar o nível de evidência dos estudos selecionados, foram utilizadas as categorias da Agency for Healthcare Research and Quality, que abrangem seis níveis: nível 1: evidências resultantes da meta-análise de múltiplos ensaios clínicos controlados e randomizados; nível 2: evidências obtidas em estudos individuais com delineamento experimental; nível 3: evidências de estudos quase experimentais; nível 4: evidências de estudos descritivos (não experimentais) ou abordagem qualitativa; nível 5: evidências de relatos de caso ou experiência; nível 6: evidências baseadas em opiniões de especialistas.

Por se tratar de uma revisão integrativa da literatura e construção de algoritmo, este estudo não necessitou da aprovação de Comitê de Ética em Pesquisa, contudo foram considerados aspectos éticos como a citação dos autores dos artigos selecionados.

A partir desse levantamento foi elaborada a cartilha, que compreende uma sequência descrita em quatro etapas, com os tópicos: avaliação clínica, classificação do tipo de LF, medidas preventivas e conduta terapêutica.

A primeira etapa descreve as etapas da avaliação clínica e, nesse momento, a cartilha educativa oferece os itens para o exame integral detalhado das condições da pele do paciente, fatores de risco associados que o paciente apresenta para contrair a LF, condições da pele adjacente, edema, coloração, aspecto da equimose, se a pele apresenta retalho, se apresenta aspecto pálido, opaco ou escurecido, presença de sangramento, tipo de tecido, presença de exsudato e faz a mensuração da lesão.

A segunda etapa classifica o tipo de LF. Foi utilizada a versão em português do "Sistema de Classificação STAR Lesão por Fricção”. Esse instrumento é constituído de três tópicos relacionados aos cuidados com a lesão e a pele ao redor, os quais vêm descritos a seguir3,15,16.

$\mathrm{Na}$ LF tipo I não ocorre perda de pele ou do retalho. Durante o curativo, o profissional poderá fazer o reposicionamento (da pele ou do retalho) para cobrir o leito da ferida.

A LF tipo II (perda parcial do retalho) apresenta perda parcial da pele ou retalho; a pele não pode ser reposicionada, ou seja, cobrir a lesão.

Na LF tipo III (perda total do retalho), o retalho de pele está completamente ausente, ou seja, a lesão está exposta, sem proteção.

$\mathrm{Na}$ terceira etapa, foram padronizados os cuidados preventivos, por exemplo: uso de emolientes ou umectantes hipoalergênicos para a lubrificação da pele, técnica de transferências, mobilização, mudança de decúbito e transferência do paciente de um leito/maca para outro a fim de evitar ou minimizar as forças de fricção, cisalhamento, contusões e torção, técnica de colocação e retirada de adesivos e utilização de sabonetes emolientes, com $\mathrm{pH}$ neutro e/ou com Aloe vera, com a recomendação de nunca utilizar sabonetes alcalinos, antibacterianos ou com perfume.

A quarta etapa indica as condutas terapêuticas para tratar a LF. Nessa fase, são descritos os cuidados locais com a pele e com a lesão e o curativo ideal, que tem como objetivo promover a cicatrização da lesão e diminuir a dor, e que é facilmente retirado, atuando como uma barreira de proteção contra a invasão bacteriana.

\section{Terceira fase: formulação/ montagem da cartilha}

As ilustrações e o conteúdo preliminares foram desenvolvidos e submetidos ao processo de edição e diagramação, obedecendo aos critérios relativos ao conteúdo, à estrutura/ organização, linguagem, ao layout e design, à sensibilidade cultural e adequação ao público idoso. Esse processo foi executado por profissional com experiência na área. 
As imagens foram selecionadas da internet e, em seguida, convertidas em desenho e trabalhadas no programa Corel Draw ${ }^{\circledR}$, versão 17. As fotos foram autorizadas pelos pacientes, originando a primeira versão da cartilha educativa submetida à validação, a qual foi elaborada no período de março a maio de 2019. A segunda versão foi construída entre os meses de setembro e dezembro de 2019.

\section{Quarta fase: validação e implementação da cartilha educativa para prevenir e tratar de lesões por fricção}

A validação de conteúdo da cartilha educativa foi efetuada com enfermeiros que atuam no tratamento de feridas no Hospital das Clínicas Samuel Libânio, com enfermeiros estomaterapeutas e enfermeiros especialistas em dermatologia.

Os critérios de inclusão dos avaliadores foram: ter certificado de curso de graduação em enfermagem com experiência mínima de um ano em tratar de pacientes com lesões cutâneas. Os critérios de exclusão dos avaliadores referiram-se aos profissionais que aceitaram participar da pesquisa, porém não responderam e/ou submeteram o questionário de avaliação no prazo de 15 dias.

Foi enviada a carta-convite para 48 profissionais, no entanto somente 32 responderam às questões do instrumento em 15 dias.

A carta-convite foi composta por: apresentação pessoal inicial e elucidações sobre o tema da pesquisa, questionário para ser avaliado pelos juízes, parecer do Comitê de Ética em Pesquisa e o Termo de Consentimento Livre e Esclarecido. Foi pormenorizado o passo a passo das etapas para a efetiva participação dos avaliadores como também informado o prazo de 15 dias para cada rodada da avaliação, a contar do dia de entrega, para efetuar e encaminhar as respostas.

Para a validação da cartilha educativa foi desenvolvido um questionário pelo próprio autor, que foi dividido em 2 partes: identificação do avaliador, com 4 questões e avaliação da cartilha educativa, com 16 questões.

Os juízes analisaram os seguintes itens da cartilha educativa: conteúdo temático, apresentação gráfica, sequência, clareza e compreensão das informações, definição de LF, tipos de categorias, fatores de risco, coberturas utilizadas nas diferentes categorias e medidas preventivas.

A escala de Likert foi utilizada nas questões de avaliação da cartilha educativa, tendo como opções de respostas: "totalmente adequada", "adequada", "parcialmente adequada", "inadequada" e "não se aplica".

A técnica Delphi foi adotada para a validação da cartilha educativa. Esse método tem como característica a obtenção de opiniões dos avaliadores com conhecimento na área por meio do uso de questionários, em que os conteúdos dos instrumentos são analisados e julgados pelos juízes em busca de um consenso (50 a 100\%) entre os avaliadores. Nesta pesquisa, optou-se por $100 \%$ de consenso entre os avaliadores.

Os dados foram compilados em uma planilha do programa Microsoft ${ }^{\circledR}$ Office 365, versão 1812, e, após codificação e tabulação, analisados por meio de estatística descritiva. As sugestões dos especialistas acatadas pelos pesquisadores foram acrescentadas na versão final da cartilha educativa.

Recorreu-se ao Índice de Validade de Conteúdo com a finalidade de medir a proporção ou porcentagem de juízes que estavam em concordância sobre determinados aspectos do instrumento e de seus itens. O valor do Índice de Validade de Conteúdo para a validação de um questionário é calculado com a soma do número de conceitos, "adequada" e "totalmente adequada", dividido pelo número total de respostas. O valor do Índice de Validade de Conteúdo deve ser maior ou igual a 0,80 .

Este estudo foi aprovado pelo Comitê de Ética em Pesquisa da Faculdade de Ciências da Saúde Dr. José Antônio Garcia Coutinho, da Universidade do Vale do Sapucaí (UNIVÁS), Pouso Alegre - MG, sob o parecer Consubstanciado número 1.426.916.

\section{RESULTADOS}

\section{Revisão integrativa da literatura}

Identificaram-se, inicialmente, 2.977 artigos. Desses, 503 foram excluídos por estarem duplicados nas bases de dados. Assim, foram selecionados 1.008 artigos para leitura do título e 43 para leitura do resumo, que resultou numa amostra de 23 artigos para a leitura do texto completo. Desses, 5 foram excluídos por não responderem à questão orientadora, o que levou ao total de 18 artigos selecionados para construir a cartilha educativa, conforme exposto na Fig. 1.

A Tabela 1 apresenta os artigos selecionados durante a revisão integrativa da literatura para desenvolver a cartilha educativa, os quais foram classificados segundo o nível em evidência. 
Artigos identificados por meio de pesquisa na base de dados $(\mathrm{n}=2.977)$

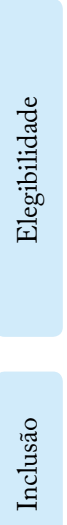

\begin{tabular}{|cll}
\hline Registros após eliminar os estudos duplicados $(\mathrm{n}=2.474)$ & $\longrightarrow$ Estudos excluídos $(\mathrm{n}=503)$ \\
\hline Estudos selecionados após a leitura do título $(\mathrm{n}=1.008)$ & & $\rightarrow$ Estudos excluídos $(\mathrm{n}=1.466)$ \\
\hline Estudos selecionados após a leitura do resumo $(\mathrm{n}=43)$ & & Estudos excluídos $(\mathrm{n}=965)$ \\
\hline Estudos selecionados após a leitura dos artigos na íntegra $(\mathrm{n}=23)$ & $\longrightarrow$ Estudos excluídos $(\mathrm{n}=20)$
\end{tabular}

Estudos selecionados para a construção da cartilha educativa $(\mathrm{n}=18)$

Estudos excluídos $(\mathrm{n}=5)$

Figura 1. Fluxograma do processo de identificação, seleção e inclusão dos estudos, elaborado a partir da recomendação do PRISMA. Pouso Alegre (MG), Brasil - 2020.

Tabela 1. Características dos artigos selecionados por meio da revisão integrativa da literatura para a construção da cartilha educativa. Pouso Alegre (MG), Brasil - 2020.

\begin{tabular}{|c|c|c|c|c|}
\hline & Autor & Título & Periódico/ano/volume/número & $\begin{array}{l}\text { Nível de } \\
\text { evidências }\end{array}$ \\
\hline 1 & $\begin{array}{l}\text { Lichterfeld-Kottner A, El } \\
\text { Genedy M, Lahmann N, } \\
\text { Blume-Peytavi U, Büscher } \\
\text { A, Kottner J.1 }\end{array}$ & $\begin{array}{l}\text { Maintaining skin integrity in the aged: } \\
\text { A systematic review. }\end{array}$ & $\begin{array}{l}\text { J. Int J Nurs Stud. 2020; 103: } 103509 . \\
\text { doi: } 10.1016 \text { / j.ijnurstu.2019.103509. }\end{array}$ & 04 \\
\hline 2 & $\begin{array}{c}\text { Rayner R, Carville K, Leslie } \\
\text { G, Dhaliwal SS. }\end{array}$ & $\begin{array}{l}\text { Models for predicting skin tears: A } \\
\text { comparison. }\end{array}$ & $\begin{array}{l}\text { Int Wound J. } 2020 \text { Jun;17(3):823-830. } \\
\text { doi: } 10.1111 / \text { iwj.13340. }\end{array}$ & 04 \\
\hline 3 & $\begin{array}{c}\text { da Silva CVB, Campanili } \\
\text { TCGF, Freitas NO, LeBlanc } \\
\text { K, Baranoski S, Santos } \\
\text { VLCG. }{ }^{3}\end{array}$ & $\begin{array}{l}\text { Istap classification for skin tears: } \\
\text { Validation for Brazilian Portuguese. }\end{array}$ & $\begin{array}{c}\text { Int Wound J. } 2020 \text { Abr;17(2):310-316. } \\
\text { doi: 10.1111/iwj.13271. }\end{array}$ & 03 \\
\hline 4 & $\begin{array}{l}\text { Scheele } \mathrm{CM} \text {, Göhner W, } \\
\text { Schumann } \mathrm{H}^{4}\end{array}$ & $\begin{array}{l}\text { Cross-sectional study on skin tears in } \\
\text { fragile, aging skin: nursing deficits in a } \\
\text { common problem in Germany. }\end{array}$ & $\begin{array}{l}\text { The Pflege. 2020; } 33 \text { (3): 123-132. } \\
\text { doi: } 10.1024 \text { / 1012-5302 / a000742. }\end{array}$ & 03 \\
\hline 5 & $\begin{array}{c}\text { Langemo DK, Williams A, } \\
\text { Edwards K. }{ }^{5}\end{array}$ & $\begin{array}{c}\text { Skin tears: prevention and } \\
\text { management. }\end{array}$ & $\begin{array}{c}\text { Nursing. } 2019 \text { Abr; } 49 \text { (4): } \\
\text { 66-69. doi: 10.1097/01. } \\
\text { NURSE.0000554309.45660.ca }\end{array}$ & 04 \\
\hline 6 & $\begin{array}{c}\text { Rayner R, Carville K, Leslie } \\
\text { G, Dhaliwal SS. }{ }^{6}\end{array}$ & $\begin{array}{l}\text { A risk model for prediction of skin } \\
\text { tears in aged care residents: a } \\
\text { prospective cohort study. }\end{array}$ & $\begin{array}{c}\text { Int Wound J. } 2019 \text { Feb; } 16 \text { (1): 52-63. } \\
\text { doi: } 10.1111 \text { / iwj.12985. }\end{array}$ & 03 \\
\hline 7 & Brown $A^{7}$ & $\begin{array}{l}\text { Skin tears: Prevention and } \\
\text { management in the elderly }\end{array}$ & $\begin{array}{l}\text { J Community Nurs 2019: } \\
\text { 33(1):p22-28. }\end{array}$ & 04 \\
\hline 8 & $\begin{array}{c}\text { Campbel K, LeBlanc K, } \\
\text { Woo K }\end{array}$ & $\begin{array}{l}\text { Best practice recommendations for } \\
\text { prevention and management of skin } \\
\text { tears in aged skin: an overview. }\end{array}$ & $\begin{array}{l}\text { Wounds International. } \\
\text { 2018; } 9 \text { (3):66-70. 10.1097/ } \\
\text { WON.0000000000000481 }\end{array}$ & 04 \\
\hline 9 & McInulty L9 & $\begin{array}{l}\text { Prevention and management of skin } \\
\text { tears in older people. }\end{array}$ & $\begin{array}{l}\text { Emerg Nurse. } 2017 \text { Jun 15;25(3):32- } \\
\text { 39. doi: } 10.7748 / \text { en.2017.e1687. }\end{array}$ & 04 \\
\hline
\end{tabular}


Tabela 1. Continuação...

\begin{tabular}{|c|c|c|c|c|}
\hline & Autor & Título & Periódico/ano/volume/número & $\begin{array}{l}\text { Nível de } \\
\text { evidências }\end{array}$ \\
\hline 10 & $\begin{array}{l}\text { Brandão CMAG, Gambin } \\
\text { CC; Majado CA, Kunitake } \\
\text { N, AlexandreNMC, Dantas } \\
\text { SRPE } 15\end{array}$ & $\begin{array}{l}\text { Adaptação do instrumento } \\
\text { "Perineal Assessment Tool” para a } \\
\text { cultura brasileira. }\end{array}$ & $\begin{array}{c}\text { ESTIMA, Braz. J. Enterostomal } \\
\text { Ther.16:e0618. } \\
\text { doi:10.30886/estima.v16.397_PT. }\end{array}$ & 03 \\
\hline 11 & $\begin{array}{l}\text { Torres FS, Blanes L, Freire } \\
\text { Galvão T, Masako Ferreira L }{ }^{16}\end{array}$ & $\begin{array}{l}\text { Development of a manual for the } \\
\text { prevention and treatment of skin tears. }\end{array}$ & $\begin{array}{l}\text { Wounds. 2018.31(1):26- } \\
\text { 32. doi: } 10.1097 / 01 \\
\text { ASW.0000405316.99011.95. }\end{array}$ & 04 \\
\hline 12 & $\begin{array}{c}\text { Tristão FR, Girondi JBR, } \\
\text { Hammerschmidt KSA, } \\
\text { Soares CF, Martins T, Lima } \\
\text { DKS. }{ }^{17}\end{array}$ & $\begin{array}{l}\text { Risco para lesão por fricção em } \\
\text { idosos longevos na atenção primária } \\
\text { à saúde. }\end{array}$ & $\begin{array}{l}\text { ESTIMA, Braz. J. Enterostomal Ther., } \\
\text { 16: e3218. https://doi.org/10.30886/ } \\
\text { estima.v16.614_PT. }\end{array}$ & 04 \\
\hline 13 & $\begin{array}{l}\text { Serra R,Lelapi N, Barbetta } \\
\text { A, De Francisci S }\end{array}$ & $\begin{array}{l}\text { Skin tears and risk factors } \\
\text { assessment: a systematic review on } \\
\text { evidence-based medicine. }\end{array}$ & $\begin{array}{l}\text { Int Wound J. 2018;15(1):38-42. } \\
\text { https://doi.org/10.12968/ } \\
\text { bjen.2019.24.Sup9.S12 }\end{array}$ & 02 \\
\hline 14 & $\begin{array}{l}\text { Koyano Y, Nakagami G, lizaka } \\
\text { S, Sugama J, Sanada } H^{19}\end{array}$ & $\begin{array}{c}\text { Skin property can predict the } \\
\text { development of skin tears among } \\
\text { elderly patients: a prospective } \\
\text { cohort study. }\end{array}$ & $\begin{array}{l}\text { Int Wound J. } 2017 \text { Aug; } 14 \text { (4): } 691 \text { - } \\
\text { 697. doi: 10.1111 / iwj.12675. }\end{array}$ & 03 \\
\hline 15 & Candeloro G. ${ }^{20}$ & $\begin{array}{l}\text { Skin tears. A quantitative study on } \\
\text { the phenomenon and proposal } \\
\text { for a prevention and management } \\
\text { procedure. }\end{array}$ & $\begin{array}{l}\text { Journal of Wound Care 2017; } \\
\qquad 1(2): 61-66\end{array}$ & 04 \\
\hline 16 & Benbow M. ${ }^{21}$ & $\begin{array}{l}\text { Assessment, prevention and } \\
\text { management of skin tears. }\end{array}$ & $\begin{array}{c}\text { Nursing of the elderly. } 2017 \text { Apr } \\
\text { 28; } 29 \text { (4): 31-39. doi: } 10.7748 \text { / } \\
\text { nop.2017.e904. }\end{array}$ & 04 \\
\hline 17 & LeBlanc K, Baranoski S.22 & Skin Tears: Finally Recognized. & $\begin{array}{l}\text { 2017;30(2):62-63. doi: 10.1097/01. } \\
\text { ASW.0000511435.99585.0d }\end{array}$ & 04 \\
\hline
\end{tabular}

A versão final da cartilha educativa denominada "Lesão por fricção: conceitos, medidas preventivas e conduta terapêutica”, contém 45 páginas, incluindo a capa, contracapa com a ficha catalográfica e o International Standard Book Number 9788567647-64-7, lista de ilustrações, lista de quadros, sumário, prefácio, apresentação e 3 capítulos (introdução, anatomia e fisiologia da pele e lesão por friç̧ão). O capítulo "lesão por fricção" foi subdividido em: definição, fatores de risco, classificação, medidas preventivas e condutas terapêuticas para a LF. Constam dois algoritmos, sendo um com medidas preventivas e outro com as condutas terapêuticas e referências bibliográficas, em tamanho padrão de formatação, com $21 \mathrm{~cm}$ de altura por $15 \mathrm{~cm}$ de largura, de acordo com a imagem na Fig. 2 .

Cada página conta com até cinco ilustrações, totalizando 22 ilustrações, que podem ser vistas na Fig. 2. A cartilha está disponível gratuitamente somente no link: http://www. univas.edu.br/menu/biblioteca/publicacoes.asp

\section{Versão final da Cartilha educativa}

$\mathrm{Na}$ Tabela 2, observamos que os juízes, durante a avaliação do conteúdo da cartilha, utilizaram a Técnica de Delphi. $\mathrm{Na}$ primeira rodada, os juízes avaliaram os conteúdos da cartilha com conceitos como: "parcialmente adequados" a "totalmente adequados".

$\mathrm{Na}$ Tabela 3, observa-se que na primeira avaliação da cartilha educativa não houve consenso entre os avaliadores nas questões: descrição da definição da categoria 1a e 1b, categoria 2a e 2b e categoria 3 da LF, e o Índice de Validade de Conteúdo variou entre 0,77 e 0,81 . Após efetuar os ajustes nas questões (descrição da definição de LF tipo I, descrição da definição de LF tipo II, descrição da definição de LF tipo III), os juízes reavaliarem a cartilha, havendo consenso entre eles. O Índice de Validade de Conteúdo foi 1,0. Esse resultado indica que o conteúdo da cartilha educativa é excelente. 


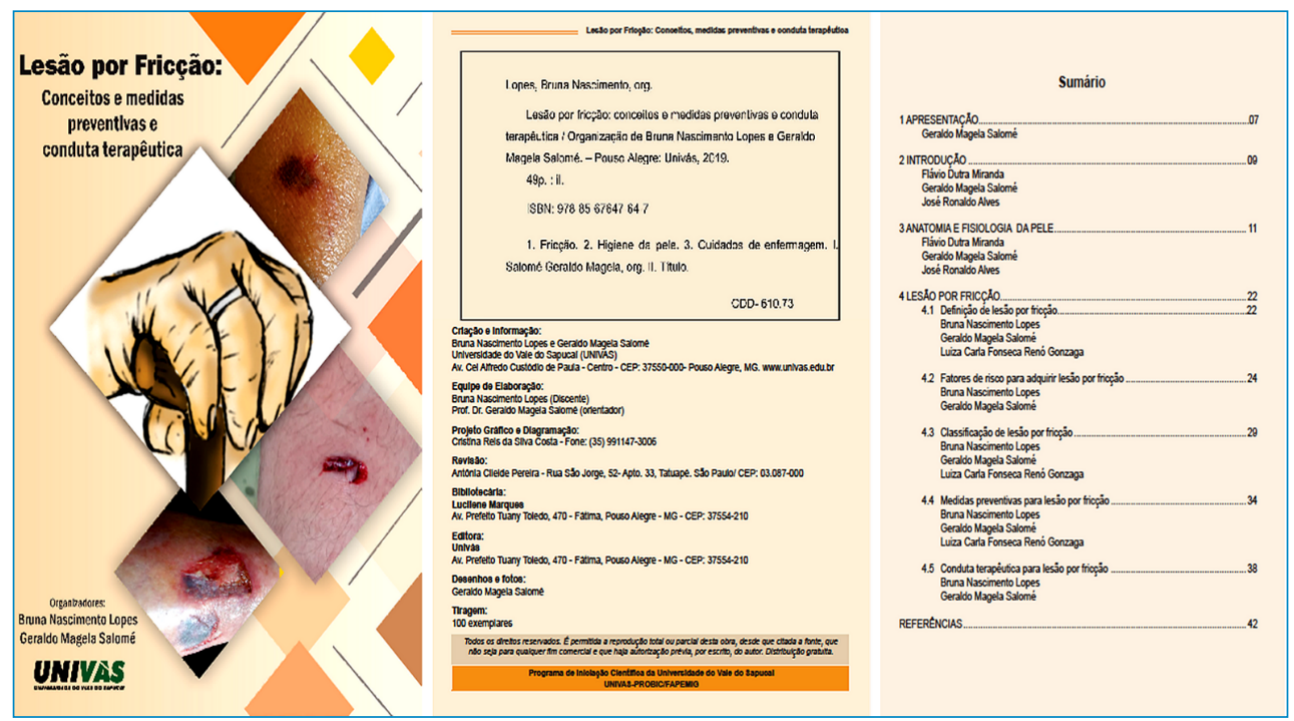

Figura 2. Capa, ficha catalográfica e sumário da cartilha Lesão por Fricção: conceitos, medidas preventivas e conduta terapêutica. Pouso Alegre (MG), Brasil - 2020.

Tabela 2. Avaliação do conteúdo da cartilha educativa pelos juízes, segundo a Técnica de Delphi. Pouso Alegre (MG), Brasil - 2020.

\begin{tabular}{|c|c|c|c|c|c|}
\hline \multirow{3}{*}{ Questões } & Inadequado & $\begin{array}{l}\text { Parcialmente } \\
\text { adequado }\end{array}$ & Adequado & $\begin{array}{l}\text { Totalmente } \\
\text { adequado }\end{array}$ & Total \\
\hline & \multicolumn{5}{|c|}{ Primeira avaliação } \\
\hline & $N(\%)$ & $\mathrm{N}(\%)$ & $N(\%)$ & $N(\%)$ & $\mathrm{n}(\%)$ \\
\hline Apresentação gráfica & $00(0,0)$ & $00(0,0)$ & $07(21,875)$ & $25(78,125)$ & $32(100)$ \\
\hline Facilidade de leitura & $00(0,0)$ & $00(0,0)$ & $05(15,625)$ & $27(84,375)$ & $32(100)$ \\
\hline Conteúdo temático da cartilha & $00(0,0)$ & $00(0,0)$ & $08(25,00)$ & $24(75,00)$ & $32(100)$ \\
\hline Vocabulário & $00(0,0)$ & $00(0,0)$ & $01(3,125)$ & $31(96,875)$ & $32(100)$ \\
\hline Clareza e compreensão das informações & $00(0,0)$ & $00(0,0)$ & $01(3,125)$ & $31(96,875)$ & $32(100)$ \\
\hline Definição de lesão por friç̧ão & $00(0,0)$ & $00(0,0)$ & $03(9,375)$ & $29(90,625)$ & $32(100)$ \\
\hline Descrição dos fatores de risco para lesão por fricção & $00(0)$ & $01(3,125)$ & $03(9,375)$ & $28(87,500)$ & $32(100)$ \\
\hline $\begin{array}{l}\text { Descrição da definição da categoria } \\
1 \text { 1a e 1b da lesão por fricção }\end{array}$ & $06(18,75)$ & $06(18,75)$ & $06(18,750)$ & $14(43,75)$ & $32(100)$ \\
\hline Descrição categoria $2 \mathrm{a}$ e $2 \mathrm{~b}$ da lesão por fricção & $00(0,0)$ & $01(03,125)$ & $06(18,750)$ & $25(78,125)$ & $32(100)$ \\
\hline Descrição categoria 3 da lesão por fricção & $07(21,875)$ & $05(15,625)$ & $06(18,750)$ & $14(43,750)$ & $32(100)$ \\
\hline Medidas preventivas para lesão por fricção & $00(0,0)$ & $01(03,125)$ & $05(15,625)$ & $26(81,250)$ & $32(100)$ \\
\hline Conduta terapêutica para lesão por fricção & $07(21,875)$ & $04(12,500)$ & $03(0,375)$ & $18(56,250)$ & $32(100)$ \\
\hline \multirow{2}{*}{ Questões } & \multicolumn{5}{|c|}{ Segunda avaliação } \\
\hline & $\mathrm{N}(\%)$ & $\mathrm{N}(\%)$ & $N(\%)$ & $N(\%)$ & $\mathrm{n}(\%)$ \\
\hline Apresentação gráfica & $00(0,0)$ & $00(0,0)$ & $07(21,875)$ & $25(78,125)$ & $32(100)$ \\
\hline Facilidade de leitura & $00(0,0)$ & $00(0,0)$ & $01(3,125)$ & $31(96,875)$ & $32(100)$ \\
\hline Conteúdo temático da cartilha & $00(0,0)$ & $00(0,0)$ & $03(9,375)$ & $29(90,625)$ & $32(100)$ \\
\hline Vocabulário & $00(0,0)$ & $00(0)$ & $03(15,625)$ & $17(84,375)$ & $32(100)$ \\
\hline Clareza e compreensão das informações & $00(0,0)$ & $00(0,0)$ & $01(3,125)$ & $31(96,875)$ & $32(100)$ \\
\hline Definição de lesão por fricção & $00(0,0)$ & $00(0,0)$ & $03(9,375)$ & $29(90,625)$ & $32(100)$ \\
\hline Descrição dos fatores de risco para lesão por fricção & $00(0,0)$ & $00(0,0)$ & $03(9,375)$ & $29(90,625)$ & $32(100)$ \\
\hline Descrição da definição lesão por fricção tipo I & $00(0,0)$ & $00(0,0)$ & $07(21,875)$ & $25(78,125)$ & $32(100)$ \\
\hline Descrição da definição lesão por fricção tipo II & $00(0,0)$ & $00(0,0)$ & $01(3,125)$ & $31(96,875)$ & $32(100)$ \\
\hline Descrição da definição lesão por fricção tipo ॥ & $00(0,0)$ & $00(0)$ & $03(15,625)$ & $17(84,375)$ & $32(100)$ \\
\hline Medidas preventivas para lesão por fricção & $00(0,0)$ & $00(0,0)$ & $07(21,875)$ & $25(78,125)$ & $32(100)$ \\
\hline Conduta terapêutica para lesão por fricção & $00(0,0)$ & $00(0,0)$ & $07(21,875)$ & $25(78,125)$ & $32(100)$ \\
\hline
\end{tabular}


Tabela 3. Índice de Validade de Conteúdo obtido no primeiro e no segundo ciclo de avaliação dos itens da cartilha educativa. Pouso Alegre (MG), Brasil - 2020.

\begin{tabular}{|c|c|}
\hline \multirow[t]{2}{*}{ Questões } & $\begin{array}{l}\text { Índice de Validade } \\
\text { de Conteúdo }\end{array}$ \\
\hline & Primeira avaliação \\
\hline Apresentação gráfica & 0,79 \\
\hline Facilidade de leitura & 0,81 \\
\hline Conteúdo temático da cartilha & 0,79 \\
\hline Vocabulário & 0,81 \\
\hline $\begin{array}{l}\text { Clareza e compreensão } \\
\text { das informações }\end{array}$ & 0,81 \\
\hline Definição de lesão por fricção & 0,80 \\
\hline $\begin{array}{l}\text { Descrição dos fatores de risco } \\
\text { para lesão por fricção }\end{array}$ & 0,80 \\
\hline $\begin{array}{l}\text { Descrição da definição da categoria } \\
1 \text { la e } 1 \text { b da lesão por fricção }\end{array}$ & 0,76 \\
\hline $\begin{array}{l}\text { Descrição categoria 2a e 2b da } \\
\text { lesão por fricção }\end{array}$ & 0,80 \\
\hline $\begin{array}{l}\text { Descrição categoria } 3 \text { da } \\
\text { lesão por fricção }\end{array}$ & 0,77 \\
\hline $\begin{array}{l}\text { Medidas preventivas para } \\
\text { lesão por fricção }\end{array}$ & 0,80 \\
\hline $\begin{array}{l}\text { Conduta terapêutica para } \\
\text { lesão por fricção }\end{array}$ & 0,78 \\
\hline IVC Geral & 0,81 \\
\hline \multirow[t]{2}{*}{ Questões } & $\begin{array}{l}\text { Índice de Validade } \\
\text { de Conteúdo }\end{array}$ \\
\hline & Segunda avaliação \\
\hline Apresentação gráfica & $* 1,0$ \\
\hline Facilidade de leitura & $* 1,0$ \\
\hline Conteúdo temático da cartilha & $* 1,0$ \\
\hline Vocabulário & $* 1,0$ \\
\hline $\begin{array}{l}\text { Clareza e compreensão } \\
\text { das informações }\end{array}$ & $* 1,0$ \\
\hline Definição de lesão por fricção & $* 1,0$ \\
\hline $\begin{array}{l}\text { Descrição dos fatores de risco } \\
\text { para lesão por fricção }\end{array}$ & ${ }^{*} 1,0$ \\
\hline $\begin{array}{l}\text { Descrição da definição lesão } \\
\text { por fricção tipo I }\end{array}$ & $* 1,0$ \\
\hline $\begin{array}{l}\text { Descrição da definição lesão } \\
\text { por friç̧ão tipo ॥ }\end{array}$ & $* 1,0$ \\
\hline $\begin{array}{l}\text { Descrição da definição lesão } \\
\text { por fricção tipo ॥ }\end{array}$ & $* 1,0$ \\
\hline $\begin{array}{l}\text { Medidas preventivas para } \\
\text { lesão por fricção }\end{array}$ & $* 1,0$ \\
\hline $\begin{array}{l}\text { Conduta terapêutica } \\
\text { para lesão por fricção }\end{array}$ & ${ }^{*} 1,0$ \\
\hline Facilidade de leitura & $* 1,0$ \\
\hline IVC Geral & $* 1,0$ \\
\hline
\end{tabular}

*Excelente Índice de Validade de Conteúdo; IVC = Índice de Validade de Conteúdo

\section{DISCUSSÃO}

A cartilha validada nesta pesquisa foi desenvolvida após revisão da literatura. Esse instrumento poderá auxiliar os profissionais das áreas da saúde, que cuidam de indivíduos que apresentam fatores de risco ou adquiriram a LF, na avaliação clínica, oferecendo medidas preventivas e condutas terapêuticas para promover a cicatrização da LF.

Uma cartilha educativa é um método eficaz para auxiliar no processo de ensino-aprendizagem na saúde e na prática clínica do profissional, de modo a aumentar sua autonomia, possibilitando melhores condutas clínicas ${ }^{11,12}$.

Vários estudos preconizam que as cartilhas, manuais e protocolos devem ser construídos após a revisão da literatura, assim o profissional estará desenvolvendo uma tecnologia educativa baseada em subsídios científicos, facilitando a implementação na prática clínica, a prestação da assistência sistematizada, individualizada e personalizada, com menor risco ao paciente, sem danos e eventos adversos ${ }^{11,12,23}$.

O material educativo no formato impresso é de fácil manuseio e pode ser levado para o domicílio, fortalecendo as orientações e sanando dúvidas sempre que necessário. $\mathrm{O}$ material escrito tem funções, tais como reforçar as informações e discussões orais e auxiliar em casos de dúvidas posteriores e nas decisões clínicas ${ }^{24,25}$.

O material desenvolvido neste estudo traz vantagens ao profissional da saúde, pois foi construído com linguagem sempre clara e objetiva e validado por profissionais com experiência e especialistas na área. Também oferece itens que ajudam o profissional a avaliar a pele dos pacientes, identificar os fatores de risco, além de fornecer protocolo em forma de algoritmos, sendo um para a prevenção e outro o para tratamento, e de modo descritivo, incluir a nova classificação do tipo de LF, medidas preventivas e as condutas terapêuticas para a LF.

As tecnologias educativas, seja qual for a situação clínica, melhoram o conhecimento e a satisfação do público-alvo. O seu conteúdo deve ser com vocabulário simples, claro e de fácil compreensão ${ }^{26}$.

Uma tecnologia educativa, para ser eficaz e de maior alcance para o profissional, deve ser desenvolvida com uma linguagem simples, que transmita uma mensagem clara e objetiva, facilitando o entendimento e a execução do procedimento $^{11,12,27}$.

Nesta pesquisa, os juízes utilizaram a Técnica de Delphi para avaliar o conteúdo da cartilha educativa. Na primeira 
avaliação não houve consenso entre os avaliadores. Após as correções realizadas com base nos comentários dos juízes e com embasamento científico, a cartilha foi reenviada para os juízes, tendo havido consenso entre eles. $\mathrm{Na}$ primeira avaliação o Índice de Validade do Conteúdo de todas as questões foi de 0,81 . Feitas as correções sugeridas pelos avaliadores, a cartilha foi enviada para outra avaliação e o Índice de Validade de Conteúdo foi 1,0, sendo este resultado significante, uma vez que foi acima do valor mínimo de concordância estipulado de $80 \%$.

Esse resultado evidencia que o conteúdo da cartilha desenvolvida neste estudo tem o potencial de ser transmitido ao público-alvo de maneira clara, simples, objetiva e com vocabulário adequado à população, o que favorece a compreensão das informações quanto aos seus aspectos técnicos e didático-pedagógicos, não havendo (ou reduzindo) a possibilidade de interpretações errôneas.

Vários estudos que validaram o conteúdo de tecnologia educativa por meio da técnica Delphi concluíram que as sugestões dos avaliadores devem ser consideradas e incorporadas. Esse procedimento contribui para uma melhor compreensão, efetividade e implantação do material na instituição, permitindo que o público-alvo possa compreender o conteúdo do material e tenha estímulo para utilizá-1o ${ }^{11,12,14,24,27}$.

Quando uma cartilha educativa é validada por profissionais com experiência na área e o Índice de Validade de Conteúdo é acima 1,0, significa que os avaliadores consideraram relevantes as informações contidas na cartilha, o que é de extrema importância para que essa tecnologia possa ser usada como ferramenta na prática clínica e na educação em saúde. A validação científica pelo público-alvo imprime credibilidade ao material ${ }^{11,12}$.

$\mathrm{Na}$ primeira versão da cartilha desenvolvida neste estudo foram implementadas várias alterações solicitadas pelos juízes. Após os ajustes, a cartilha foi reavaliada, tendo havido consenso entre os avaliadores. Essa análise crítica foi fundamental para o aprimoramento da nova versão da cartilha educativa.

A cartilha desenvolvida tem como propósito nortear os profissionais de saúde na prática clínica, assistir os indivíduos em tempo hábil, de forma efetiva, sistematizada, individualizada, personalizada, com o mínimo possível de risco, sem danos e eventos adversos, proporcionando uma assistência com segurança.

\section{CONCLUSÃO}

O conteúdo da cartilha educativa foi construído e validado. Na primeira avaliação apresentou 0,81 de Índice de Validade de Conteúdo, significando que a cartilha apresenta bom conteúdo, tendo sido feitas várias sugestões. Após realizar as correções indicadas pelos avaliadores, a cartilha foi reavaliada e o Índice de Validade de Conteúdo foi 1,0, caracterizando que a cartilha apresenta excelente conteúdo.

A cartilha construída neste estudo oferece condições para o profissional realizar avaliação clínica, detectar os fatores de risco e prescrever medidas preventivas e condutas terapêuticas. Também facilita o registro das características da LF, assegurando o monitoramento da evolução da lesão, minimizando riscos, danos e eventos adversos.

\section{REFERÊNCIAS}

1. Lichterfeld-Kottner A, El Genedy M, Lahmann N, BlumePeytavi U, Büscher A, Kottner J. Maintaining skin integrity in the aged: a systematic review. Int J Nurs Stud 2020;103:103509. https://doi.org/10.1016/j.ijnurstu.2019.103509

2. Rayner R, Carville K, Leslie G, Dhaliwal SS. Models for predicting skin tears: A comparison. Int Wound J. 2020 Jun;17(3):823-830. https://doi.org/10.1111/iwj.13340

3. Silva CVB, Camanili TCGF, Freitas NO, LeBlanc K, Baranoski $\mathrm{S}$, Santos VLCG. ISTAP classification for skin tears: validation for brazilian portuguese. Int Wound J 2020;17(2):310-6. https://doi.org/10.1111/iwj.13271
4. Scheele CM, Göhner W, Schumann H. Cross-sectional study on skin tears in fragile, aging skin: nursing deficits in a common problem in Germany. Pflege 2020;33(3):123-32. https://doi.org/10.1024/1012-5302/a000742

5. Langemo DK, Williams A, Edwards K. Skin tears: prevention and management. Nursing 2019;49(4):66-9. https://doi. org/10.1097/01.NURSE.0000554309.45660.ca

6. Rayner R, Carville K, Leslie G, Dhaliwal SS. A risk model for prediction of skin tears in aged care residents: a prospective cohort study. Int Wound J 2019;16(1):52-63. https://doi. org/10.1111/iwj.12985 
7. Brown A. Skin tears: Prevention and management in the elderly. J Community Nurs 2019:33(1):22-8. Disponível em: https://www.researchgate.net/publication/331839054 Skin_tears_Prevention_and_management_in_the_elderly

8. LeBlanc K, Campbel KE, Wood E, Beeckman D. Best practice recommendations for prevention and management of skin tears in aged skin: an overview. J Wound Ostomy Continence Nurs 2018;45(6):540-2. https://doi.org/10.1097/ WON.0000000000000481

9. Mclnulty L. Prevention and management of skin tears in older people. Emerg Nurs 2017;25(3):32-9. https://doi. org/10.7748/en.2017.e1687

10. Catania QN, Morgan M, Martin R. Activity-Based Restorative Therapy and Skin Tears in Patients with Spinal Cord Injury. Adv Skin Wound Care 2018;31(8):371-3. https://doi. org/10.1097/01.ASW.0000534700.57785.84

11. Salomé GM, Cunha AL, Pereira AP, Miranda FD, Alves JR. Educational handbook for healthcare professionals: preventing complications and treating peristomal skin. J Coloproctol 2019;39(4):332-8. https://doi.org/10.1016/j. jcol.2019.07.005

12. Mendes B, Salomé GM, Pinheiro FAM, Júnior MRM, Cunha DR, Ferreira LM. Preventing and treating trench foot: validation of an educational manual for military personnel. J Wound Care 2018;27(Sup10):S33-8. https://doi.org/10.12968/ jowc.2018.27.Sup10.S33

13. Mendes KDS, Silveira RCCP, Galvão CM. Revisão integrativa: método de pesquisa para a incorporação de evidências na saúde e na enfermagem. Texto Contexto Enferm 2008;17(4):758-64. http://doi.org/10.1590/S010407072008000400018

14. Santos CMC, Pimenta CAM, Nobre MRC. The Pico strategy for the research question construction and evidence search. Rev Latino-Am Enfermagem 2007;15(3):508-11. https://doi. org/10.1590/s0104-11692007000300023

15. Brandão ACMAG, Gambin CC; Majado CA, Kunitake N, Alexandre NMC, Dantas SRPE. Adaptação do instrumento "Perineal Assessment Tool" para a cultura brasileira. ESTIMA, Braz J Enterostomal Ther 2018;16:e0618. https:// doi.org/10.30886/estima.v16.397_PT

16. Torres FS, Blanes L, Galvão TF, Ferreira LM. Development of a manualforthepreventionand treatmentofskintears. Wounds 2018; [citado em 01 dez 2020]; 31(1):26-32. Disponível em: https://www.biosanas.com.br/uploads/outros/artigos_ cientificos/51/adc7302e06f88ce154d9b5ee99f472aa.pdf
17. Tristão FR, Girondi JBR, Hammerschmidt KSA, Soares CF, Martins T, Lima DKS. Risco para lesão por fricção em idosos longevos na atenção primária à saúde. ESTIMA, Braz J Enterostomal Ther 2018;16:e3218. https://doi. org/10.30886/estima.v16.614_PT

18. Serra R, lelapi N, Barbetta A, Franciscis S. Skin tears and risk factors assessment: a systematic review on evidencebased medicine. Int Wound J. 2018;15(1):38-42. https://doi. org/10.1111/iwj.12815

19. Koyano Y, Nakagami G, lizaka S, Sugama J, Sanada H. Skin property can predict the development of skin tears among elderly patients: a prospective cohort study. Int Wound J 2017;14(4):691-7. https://doi.org/10.1111/iwj.12675

20. Candeloro G. Skin tears. A quantitative study on the phenomenon and proposal for a prevention and management procedure. Ital J Wound Care 2017;1(2):61-6. https://doi.org/10.4081/ijwc.2017.13

21. Benbow M. Assessment, prevention and management of skin tears. Nursing Older People 2017;29(4):31-9. https:// doi.org/10.7748/nop.2017.e904

22. LeBlanc K, Baranoski S. Skin Tears: Finally Recognized. Adv Skin Wound Care. 2017;30(2):62-63. https://doi. org/10.1097/01.ASW.0000511435.99585.0d

23. Normansell R, Kew KM, Mathioudakis AG. Interventions to improve inhaler technique for people with asthma. Cochrane Database Syst Rev 2017; 3(3):CD012286. https:// doi.org/10.1002/14651858.CD012286.pub2

24. Salomé GM, Alves NF. App "SICKSEG" in mobile platform for the prevention of skin injuries. J Nurs UFPE on line 2020;14:e244152. https://doi.org/10.5205/19818963.2020 .244152

25. Santos I, Brandão ES, Clós AC. Dermatology nursing: sensitive listening skills and technology for acting in skin care. Rev Enferm UERJ 2009; [citado 2019 ago 10]; 17(1):124-9. Disponível em: http://files.bvs.br/upload/S/0104-3552/2009/ v17n1/a022.pdf

26. Carvalho MRF, Salomé GM, Ferreira LM. Construction and validation of algorithm for treatment of pressure injury. J Nurs UFPE on line 2017;11(Suppl. 10):4171-83. https://doi. org/10.5205/reuol.10712-95194-3-SM.1110sup201722

27. Salomé GM, Rocha CA, Miranda FD, Alves JR, Dutra RAA, Tenório AG. Algoritmos para prevenção e tratamento de dermatite associada à incontinência. ESTIMA, Braz J Enterostomal Ther 2020:e1320. https://doi.org/10.30886/ estima.v18.837_IN 